\title{
ADVERBIOS EN -MENTE Y LA ESTRUCTURA DEL ADJETIVO EN ESPAÑOL
}

\author{
ANTONIO FÁBREGAS \\ Universitetet i Troms $\varnothing$ \\ webfabregas@gmail.com
}

\begin{abstract}
Resumen
En este artículo propongo un análisis sintáctico de los adverbios terminados en mente del español. Argumento que mente, relacionado históricamente con un nombre, debe analizarse en español actual como una matriz de rasgos interpretable de género y número. En este sentido, esta pieza posee las propiedades de un nombre, pero, al contrario que éstos, carece de un índice de identidad, lo cual le imposibilita para ser sujeto de una predicación. La función de -mente es la de satisfacer la concordancia del adjetivo con el que se combina sin convertirse en su sujeto; por tanto, la combinación con mente permite al adjetivo convertirse en un predicado de categorías no nominales. Dado que la función primordial de mente es la de satisfacer la concordancia del adjetivo, predecimos que los adjetivos relacionales y otros no predicativos pueden dar lugar a adverbios en -mente. Por último, nos ocupamos del problema de cómo restringir la productividad de este afijo.

PALABRAS CLAVE: Adjetivos, adverbios, concordancia, morfología, sintaxis.
\end{abstract}

\begin{abstract}
In this article I provide a syntactic analysis of Spanish adverbs ending in -mente. I argue that mente, which is diachronically related to a noun, has to be analysed in synchronic Spanish as the spell out of a matrix of interpretable features for gender and number. In this sense, mente has properties of a noun, but, in contrast with normal nouns, it lacks an index of identity, which makes it unavailable as a subject of a predicate. The function of -mente is to satisfy the agreement of the adjective with which it combines, without becoming its subject of predication; therefore, combination with -mente allows the adjective to act as a predicate of non-nominal categories. As the primary function of -mente is to satisfy the agreement of the adjective, we also predict that relational adjectives and other adjectives which are non predicative can produce adverbs in mente. Finally, we also address the problem of how to restict the productivity of this affix.
\end{abstract}

KEYWORDS: Adjectives, Adverbs, Agreement, Morphology, Syntax.

\section{Interés teórico de los $\operatorname{AdP}^{1}$}

Los adverbios con valor predicativo del español (AdP) se construyen productivamente mediante la adición de la pieza léxica -mente a un adjetivo (1). Admiten la combinación con -mente la mayor parte de los adjetivos de cualidad (cfr. Demonte, 1999 para una detallada clasificación de estos elementos) -de manera (1a), de dimensión física (1b) o de

${ }^{1}$ La investigación que subyace a este proyecto ha sido financiada mediante la beca post-doctoral del MEC EX2009-0968. 
disposición humana (1c)-; es bien sabido que, en cambio, algunos adjetivos, como los de color, no pueden construir AdP (1d).

(1) a. lent-a-mente, hermos-a-mente, cuidados-a-mente, elegant-e-mente...

$b$. hond-a-mente, alt-a-mente, enorm-e-mente, grues-a-mente...

$c$. horad-a-mente, generos-a-mente, codicios-a-mente, malign-a-mente...

d. *roja-mente, *amarilla-mente...

El adjetivo que se combina con la forma -mente aparece siempre en su forma femenina, no solo en español, sino también en francés e italiano. La explicación más simple de esta peculiaridad es que el adjetivo concuerda con femenino singular con la forma mente, que, como nombre autónomo, es un sustantivo femenino. Esta explicación, sin embargo, ha sido descartada sobre la base de problemas teóricos relacionados con la Hipótesis de la Integridad Léxica (Lapointe, 1979), que, entre otras cosas, prohíbe que se establezcan relaciones sintácticas como la concordancia entre dos constituyentes internos de palabra.

A pesar de que a menudo se ha afirmado que los adjetivos relacionales no admiten la combinación con -mente, datos como los de (2) muestran que esto no es cierto. Si bien los adverbios resultantes tienen usos distintos a los de (1), desde el punto de vista de la morfología de la palabra, la operación parece la misma y el requisito de la presencia de la forma femenina también es idéntico. Las formas resultantes no tienen valor predicativo -de igual manera que los adjetivos relacionales no lo tienen-, por lo que, si bien es cierto que los adverbios predicativos se construyen con -mente, no lo es que todos los adverbios en mente sean predicativos.

(2) polític-a-mente, económic-a-mente, artístic-a-mente...

Esta descripción es bien conocida, igual que también lo son los problemas teóricos y de análisis a los que dan lugar estas formas. Pese a ello, revisaré brevemente sus problemas más destacados.

Desde el punto de vista de la morfología, no está nada claro qué tipo de operación es la que da lugar a los adverbios en -mente. Las propuestas van desde quienes proponen que mente es una forma flexiva (Hjelmslev, 1928; Alarcos, 1951) a quienes aseguran que es un morfema derivativo (Varela, 1990; Scalise, 1993) o compositivo (Alonso y Henríquez Ureña, 1955; Seco, 1972; Zagona, 1990). Hay, también, otros autores que consideran que estas formas han sido construidas mediante procedimientos sintácticos (Alcina y Blecua, 1975; Bello, 1847; Lenz, 1935).

Ninguna de las propuestas morfológicas está exenta de problemas.

Como señala Scalise (1993), la productividad de las formas en -mente, aunque muy elevada, no es comparable a la de los procesos flexivos, que son, por definición, de naturaleza paradigmática. No es posible afirmar que todos los adjetivos de una clase tengan una forma en -mente, como muestra la agramaticalidad de las formas *rojamente o *redondamente. Asimismo, si -mente fuera un morfema flexivo, sería una excepción fonológica en su clase, pues esta clase de afijos, típicamente, están reducidos fonológicamente. 
La propuesta derivativa, aunque es la más aceptada, no carece tampoco de complicaciones. La más grave de todas es, en nuestra opinión, que, sobre la suposición de que -mente es una forma derivativa, el orden en que la marca de femenino y este morfema aparecen infringe la Hipótesis de Integridad Léxica, y también parece ir contra la generalización de que los morfemas derivativos son internos a los morfemas flexivos, lo cual parece un universal morfológico (Greenberg, 1967). El orden de (3), sin embargo, es factualmente imposible.

\section{(3) *continu-ment(e)-a}

La propuesta compositiva se enfrenta al hecho de que, típicamente, los compuestos establecen entre sus constituyentes relaciones semánticas asimilables a las relaciones de descarga temática, con el resultado de que parte de la estructura argumental de la base queda alterada cuando se somete a composición: limpia-botas, pelirrojo, etc. No obstante, Bosque (1989: 134) muestra que la adición de -mente no altera la estructura argumental de las bases con las que se combina (4).

(4) a. paralelo a esto - paralelamente a esto

b. independiente de ello - independientemente de ello

c. proporcional al resultado - proporcionalmente al resultado

Los adjetivos de (4) son diádicos y toman un complemento, como se ve en el primer miembro del par; esta capacidad seleccional no queda afectada por la adición de -mente, en contraste con lo que esperamos de un proceso compositivo.

Asimismo, las tres teorías se han de enfrentar a un problema común, que es la posibilidad de coordinar dos adjetivos en femenino que comparten una sola forma -mente. De nuevo, esta propiedad está compartida por dialectos de otras lenguas romance, como el catalán antiguo.

(5) a. [rápida- y efectiva-]mente

b. *[depila- y confec-]ción (cfr. depilación y confección)

c. [la mesa y la silla] de la clase

La peculiaridad de esta construcción queda ilustrada en el contraste de (5): dos bases morfológicas coordinadas no pueden compartir un mismo sufijo; solo los constituyentes sintácticos admiten ser coordinados y compartir un constituyente.

En este trabajo, propondremos un análisis puramente sintáctico de los adverbios en mente. El modelo teórico en el que incardinamos este análisis defiende, al igual que parte del estructuralismo, que las palabras y las oraciones se construyen mediante el mismo conjunto de reglas. No obstante, nuestro análisis es hasta cierto punto independiente de estos modelos, en la medida en que podría ser cierto que los adverbios en -mente se construyen en la sintaxis -como defiende nuestro análisis-, mientras que otras palabras con comportamiento distinto se podrían formar en el léxico. 
Nuestro análisis se cimenta, más concretamente, en los modelos teóricos según los cuales la formación de palabras tiene lugar en el componente sintáctico; de hecho, seremos más extremos que algunas de estas propuestas, como la Morfología Distribuida (cfr. Marantz, 2001 y las referencias contenidas allí), que admite la existencia de un conjunto de operaciones interpretativas que modifican la estructura sintáctica (Embick y Noyer, 2001). Aunque en el análisis que sigue no es inmediatamente relevante $\mathrm{y}$, de hecho, nuestro análisis es compatible con cualquier teoría sintáctica de la morfología, nuestras suposiciones teóricas están más cerca de las propuestas de Ramchand (2008), Caha (2007) y Michal Starke (trabajos no publicados), que diseñan sistemas en los que la morfología tiene lugar íntegramente en la sintaxis, y las diferencias superficiales en lenguas diferentes son debidas al elenco de formas léxicas de una lengua -más específicamente, a qué rasgos sintácticos materializa cada pieza-.

Un segundo componente de nuestro análisis tiene que ver con nuestra concepción de las categorías léxicas. En la línea de la Morfología Distribuida, aceptamos que las categorías gramaticales son construidas en la sintaxis y carecen de realidad independiente. Más concretamente, adoptamos una teoría restrictiva de las categorías gramaticales en las que cada una de ellas se corresponde biunívocamente con una configuración sintáctica (Hale y Keyser, 2002), de manera que no es posible saber qué categoría gramatical va a recibir una matriz de rasgos determinada antes de que dicho conjunto de rasgos sea insertado en la sintaxis y forme parte de una configuración.

La estructura del artículo es como sigue: en el punto 2, exponemos tres propiedades de los adverbios en -mente que nos parecen cruciales para su análisis. En el punto 3, presentamos su análisis en términos puramente sintácticos, y damos cuenta de sus propiedades a partir de una única estructura que integra al adverbio en una teoría general de los adjetivos. En 4, por último, mostraremos cómo este análisis permite dar cuenta de la productividad de estas formaciones sin recurrir al léxico.

\section{Tres propiedades de los AdP}

En esta sección revisaremos tres propiedades de los AdP del español que, según creo, no han recibido la atención debida y nos permiten fundamentar nuestro análisis.

\subsection{Los AdP son predicados de categorías no nominales}

La primera propiedad, tal vez la más clara, de los AdP es que son modificadores de categorías no nominales (cfr. Iglesias Bango, 2004). Los lugares donde típicamente se generan los AdP son distintos puntos del sintagma verbal ( $\mathrm{Sv}$ ), como adjuntos de manera o de resultado, entre otras nociones que afectan a distintos aspectos de la acción verbal, o del sintagma complementante (SC), en cuyo caso señalan propiedades del enunciado o de la enunciación.

Lo que tienen en común todos estos casos es que el adjetivo sobre el que se ha construido el adverbio conserva su capacidad predicativa, si la tenía. Supongamos una estructura funcional rica como la que propone Cinque (1999); dependiendo de la posición 
que ocupa el adverbio, las propiedades denotadas por el predicado se predican de la entidad referida por una categoría no nominal diferente. En (6a), completamente modifica el subevento de resultado de la acción designada por el verbo, predicando de esta fase aspectual que se halla completa; en (6b), el adverbio lamentablemente modifica al subevento de proceso del verbo, indicando que dicho evento tiene la propiedad de ser lamentable.

(6) a. Juan escribió el artículo completamente.

b. Juan toca el piano lamentablemente.

En una semántica neo-davidsoniana, estos hechos pueden representarse si entendemos que las propiedades designadas por el adjetivo se predican del rasgo categorial 'evento' (E) contenido en el propio verbo. Así se explica, como es bien sabido, que los verbos estativos (7b) no admitan adverbios orientados al verbo.

(7) a. \{comer / fumar / construir / caer / llorar \} lentamente. b. *\{gustar / tener / doler / contener \} lentamente.

Si esto es así, es posible representar la composición semántica de un adverbio orientado al verbo como se ve en (8), donde 'e' es una notación que sirve para representar cualquiera de los subeventos verbales.

(8) $\exists \mathrm{e} \lambda \mathrm{e}[\operatorname{adjectivo'}(\mathrm{e})]$

En el caso en que el adverbio predica propiedades del acto de enunciación -y, por ende, del hablante (9a)- o del enunciado mismo (9b, 9c), el adjetivo interno al AdP descarga su información semántica con la denotación del nudo C. En estos casos, se califica de franco al acto de enunciación, en el primer caso, y de posibles o necesarias a las proposiciones denotadas por la cláusula, en los dos últimos ejemplos.

(9) a. Francamente, esto no puede seguir así.

b. Posiblemente, Juan volverá a caer.

c. Necesariamente, el lobo ataca a la oveja.

La equivalencia de las oraciones de (9) con secuencias como 'El hecho de que el lobo ataque a la oveja es necesario' o 'El acto por el que afirmo que esto no puede seguir así es franco' sugiere que, al igual que sucedía en el caso anterior, es posible tratar esta clase de modificación con una fórmula semántica semejante a la que hemos introducido en (8) para los distintos subeventos verbales. Podría proponerse que el nudo complementante contiene un rasgo semántico $\mathrm{P}$ que denota una proposición completa, $\mathrm{y}$ en alguna de sus proyecciones extendidas, un rasgo $\mathrm{H}$ que ancla la proposición a un acto de habla. Si esto fuera así, la representación de las oraciones de (9) se podría representar como (10). 
(10) a. $\exists \mathrm{P} \lambda \mathrm{P}[$ [adjectivo' (P)]

b. $\exists \mathrm{H} \lambda \mathrm{H}$ [adjectivo' $(\mathrm{H})]$

Naturalmente, estas representaciones semánticas son muy rudimentarias e ignoran conscientemente numerosos aspectos de la representación formal de las proposiciones y los actos de habla, pero lo que resulta relevante para nuestros propósitos es que el adverbio funciona como un elemento que predica de un constituyente que carece de categoría nominal las propiedades denotadas por el adjetivo sobre el que se construye.

Como vemos aquí, nada impide que un adjetivo se predique semánticamente de un constituyente no nominal, como un verbo o una cláusula completa. Lo que privilegia la relación predicativa del adjetivo con el sustantivo no es, pues, un factor semántico, sino más bien morfológico: el adjetivo, en una lengua como el español, contiene rasgos que exigen la concordancia con el sustantivo en número y persona, y categorías como el sintagma verbal y el sintagma complementante carecen de estos rasgos. En este trabajo propondremos que precisamente el procedimiento con el que cuenta el español para satisfacer la concordancia del adjetivo y permitirle actuar como predicado de una categoría no nominal es la afijación con-mente.

\subsection{El constituyente-mente no altera la semántica del adjetivo}

La segunda propiedad relevante de los AdP es que -mente no aporta ninguna semántica a la construcción. No solo eso, sino que, además, la presencia de -mente no impide que la semántica del adjetivo sobre el que se construye el adverbio sea accedida completamente por el componente semántico. Las pruebas provienen del comportamiento de los adverbios en -mente como modificadores del sintagma verbal y de la correspondencia biunívoca entre la denotación de los adjetivos sobre los que se construyen y las posibilidades combinatorias del adverbio correspondiente con distintas clases aspectuales de verbos. Rodríguez Ramalle (2002) identifica dos clases de adjetivos. La primera de ellas designa propiedades de los individuos, y admite ser recategorizada como la manera en la que dichos individuos desempeñan determinadas acciones (11a). La segunda clase de adjetivos no se puede predicar de individuos, sino de las circunstancias que rodean la realización de distintos sucesos (11b).

(11) a. brillante, estupendo, cuidadoso, rápido, hábil... b. casual, accidental, definitivo, imprevisto...

Los adverbios construidos sobre el grupo de (11a) solo pueden predicarse de acciones en su desarrollo, es decir, de procesos, pues es en el decurso de un proceso cuando los individuos manifiestan sus propiedades; en cambio, los adverbios construidos sobre (11b) solo pueden predicarse sobre el subevento que denota la culminación de una acción, pues es entonces cuando se puede caracterizar el modo en que ha resultado dicha acción. Esto explica la distribución de (12) y (13): cuidadosamente no puede modificar a verbos como encontrar, pues carecen de un subevento procesual, mientras que accidentalmente no puede 
modificar a la clase de buscar, pues estos verbos no contienen un subevento de resultado en el que culmine la acción.

(12) encontrar algo, detectar algo, alcanzar algo...

a. *detectar el cáncer \{cuidadosamente / concienzudamente

b. detectar el cáncer \{accidentalmente / imprevistamente\}

(13) buscar algo, perseguir algo, correr...

a. buscar el lápiz \{cuidadosamente / concienzudamente\}

b. *buscar el lápiz \{accidentalmente / imprevistamente\}

La sistematicidad de esta correspondencia no sería fácil de entender si -mente aportara alguna noción predicativa de alguna clase al AdP. Más allá de esto, datos como los de (12) y (13) son incompatibles con un análisis en que -mente realice un rasgo como 'orientado al verbo' (Kornfeld, 2006). Como se puede ver, la presencia de -mente no faculta per se al adjetivo para poder orientarse al verbo: es el adjetivo el que determina si dicha orientación es posible, o no, y con qué verbos.

\subsection{Hay un adjetivo pleno en el interior del adverbio}

Es posible mostrar de forma directa que la presencia de -mente no cambia la categoría del adjetivo, y que éste sigue exhibiendo todas las propiedades formales que exhibía como adjetivo independiente.

Dos son las propiedades morfológicas características de los adjetivos en español. La primera de ellas es que los adjetivos concuerdan, y ya hemos visto que, desde una perspectiva libre de prejuicios acerca del comportamiento de la morfología, los AdP del español parecen exhibir concordancia en femenino singular con -mente. Si no se desea estipular que, por alguna razón idiosincrática, la forma -mente exige la presencia de una forma femenina del adjetivo, la explicación más directa es, en nuestra opinión, admitir que la forma aparece en femenino porque concuerda de alguna forma con -mente, lo cual, además, es compatible con lo que sabemos de la evolución histórica de la forma.

Técnicamente, la concordancia es el resultado superficial de un proceso sintáctico de borrado de rasgos no interpretables. Como se sabe (Chomsky, 1995; Eguren y Fernández Soriano 2004), en el Programa Minimista, los elementos sintácticos son conjuntos de rasgos, y la sintaxis es el componente en que dichos rasgos interactúan entre sí. Los rasgos se dividen en dos clases: interpretables y no interpretables. Si los primeros entran en la sintaxis con un valor asignado, el segundo tipo de rasgos carece de valor, por lo que no pueden ser interpretados por los componentes sintáctico y fonológico. Por ello, para que la estructura sea gramatical, estos rasgos han de desaparecer de la representación, y la forma de conseguir que desaparezcan es mediante concordancia con un conjunto de rasgos interpretables. La concordancia, pues, queda así capturada como un procedimiento por el que rasgos sin valor copian en la sintaxis el valor de los rasgos que ya lo poseían. Por conveniencia expositiva, representaremos los rasgos sin valor anteponiendo a su nombre el signo $\alpha$. 
En el caso particular de los adjetivos, parece claro que los rasgos no interpretables que contienen son [ $\alpha$ Género] y [ $\alpha$ Número], pues la concordancia con el sustantivo requiere precisamente estos dos accidentes morfológicos. Por brevedad, representaremos el género con la abreviatura Gen y el número como \#. [ $\alpha \mathrm{Gen}]$ y [ $\alpha \#]$, contenidos en el adjetivo, deben concordar con una categoría que posea [Gen] y [\#], es decir, los mismos rasgos, pero en versión interpretable. Característicamente, la categoría que contiene estos dos rasgos interpretables en español es el sustantivo, lo cual explica que los adjetivos escojan privilegiadamente a los sustantivos para establecer relaciones sintácticas con ellos: los necesitan para satisfacer la necesidad de concordar en número y género.

En español se comprueba que el sintagma nominal con el que se establece la concordancia es también el constituyente del que se predican las propiedades denotadas por el adjetivo. La oración de (14) ilustra esta propiedad: cuando el predicativo concuerda en femenino, se interpreta que la fugitiva estaba exhausta; cuando lo hace en masculino, la lectura es que es el policía quien lo estaba.

\section{(14) El policía perseguía a la fugitiva \{exhausto / exhausta\}}

Esto nos lleva a la pregunta de si la capacidad predicativa del adjetivo -que, como vimos en 2.1, conserva el adverbio correspondiente- debe representarse mediante algún rasgo. Algunos autores, como Spencer (1999), proponen caracterizar al adjetivo con un rasgo semántico [Atribución] -[Atr] a partir de ahora- que se coindiza con el índice referencial del sustantivo para poder establecer la relación de predicado-argumento. En el tercer apartado volveremos sobre esta cuestión, y propondremos que la correlación entre concordancia y predicación, ilustrada en (14), tiene su explicación en términos configuracionales. La idea que desarrollaremos es que, para establecer la concordancia con el adjetivo, el nombre debe desplazarse a cierta posición sintáctica, en la que necesariamente debe ser interpretado como el sujeto de la predicación.

La segunda propiedad morfológica de los adjetivos es la gradabilidad: los adjetivos predicativos en español aceptan, por lo general, el morfema superlativo -ísimo. Esta propiedad también la manifiestan los adjetivos que son bases de los AdP: cuando el adjetivo admite por separado morfología de grado, también la admite el adverbio correspondiente (15).

(15) a. rápid-o - rapid-ísim-o

b. rápid-a-mente - rapid-ísim-a-mente

Se ha observado en la literatura semántica que los adjetivos son constituyentes cuyo valor de verdad solo puede ser determinado por referencia a una escala cuya medida estándar se fija, en ausencia de otras indicaciones, contextualmente (Kamp, 1975; Cresswell, 1976; Kennedy, 1999). Por esta razón, algunos autores más preocupados por la sintaxis han argumentado (Zwaarts, 1992) que los adjetivos contienen un papel semántico de grado y han de estar siempre dominados por una proyección sintáctica con dicha información. Una forma de representar técnicamente esta propiedad semántica es dotar a los 
adjetivos de un rasgo no interpretable [ $\alpha$ Grado] o $[\alpha G]$ que les obliga a entrar en concordancia con una proyección que contenga [G]. Seguiré la tradición (cfr., por ejemplo Zwaarts, 1992; Corver, 1997) y llamaré a dicho sintagma Sintagma Grado o SG.

En consecuencia, los rasgos que parecen definir a los adjetivos en español son los representados en (16), y, como hemos visto, hay razones directas para pensar que estos mismos rasgos se encuentran presentes en el interior de los AdP, por lo que concluimos que estos adverbios contienen un genuino adjetivo en su interior.

(16) $[\alpha \mathrm{Gen}, \alpha \#, \alpha \mathrm{G}, \mathrm{Atr}]$

En la siguiente sección propondremos un análisis de estas construcciones en términos sintácticos.

\section{Una morfología sintáctica para los AdP}

En esta sección argumentaremos que la existencia de AdP, la elección particular de los lexemas implicados y la estructura general del adverbio como palabra se derivan de principios gramaticales independientes. En nuestro análisis emplearemos configuraciones jerárquicas de relaciones sintácticas, aunque creemos que nuestras intuiciones pueden ser recogidas en cualquier teoría que admita que existen constituyentes internos a la palabra que tienen funciones sintácticas determinadas.

La argumentación que desarrollaremos es la siguiente. Los adjetivos en español requieren concordancia en género y número, lo cual hace que establezcan relaciones sintácticos con los nombres. Un resultado inmediato del establecimiento de esta relación sintáctica es que el nombre se convierte en el sujeto de predicación del adjetivo. Esto impediría, en principio, que un adjetivo se predicara de cualquier categoría no nominal. Sin embargo, si existe un elemento que contiene los rasgos de género y número que el adjetivo necesita, pero que, por motivos independientes, no puede ser el sujeto de predicación del adjetivo, entonces el adjetivo, habiendo satisfecho sus requisitos de concordancia, y teniendo libre su capacidad predicativa, puede tomar como sujeto de predicación a una categoría no nominal, como el verbo o el complementante. Proponemos que un elemento así, con rasgos de género y número e incapaz de ser sujeto de predicación, existe, y es precisamente -mente, de tal forma que, cuando éste se combina con el adjetivo para dar lugar a lo que la tradición ha llamado 'adverbio', dicho adjetivo puede predicarse de categorías no nominales.

\subsection{La estructura sintáctica de los adjetivos predicativos del español}

Defenderemos que la estructura sintáctica que comparten adjetivos y AdP en español es la de (17). En este árbol hemos representado los rasgos relevantes: rasgos no interpretables de género, número y grado en el sintagma adjetivo (SA), rasgos interpretables de grado en el $\mathrm{SG}$ y rasgos interpretables de género y número en el sintagma nominal (SN). 
(17)

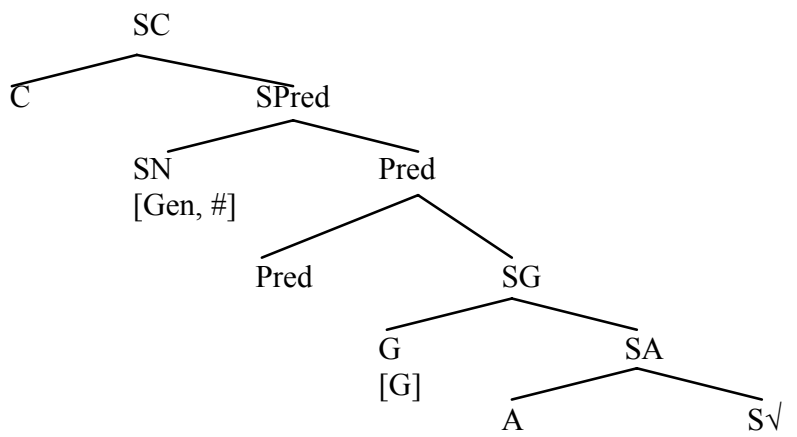

$[\alpha \mathrm{Gen}, \alpha \#, \alpha \mathrm{G}, \mathrm{Atr}]$

En las siguientes subsecciones, iremos motivando cada uno de los pasos de esta estructura. Nótese, sin embargo, que en (17) estamos empleando una proyección cuya etiqueta es raíz $(\sqrt{ })$. Esta proyección representa un nudo sintáctico que carece por sí misma de categoría léxica y sólo recibe una contextualmente. Creemos que existen pruebas independientes de la existencia de esta proyección -por ejemplo, la conversión (Fábregas, 2005) y los truncamientos (Marantz, 2001)-, aunque en lo que sigue, nuestro análisis no depende de forma crucial de la existencia de este nudo y podría mantenerse incluso en el caso de que no hubiera proyecciones sintácticas sin categoría léxica.

\subsubsection{La sintaxis de los adjetivos predicativos}

En su análisis de las diferencias entre las categorías gramaticales, Hale y Keyser (2002) observan que los adjetivos son categorías que, desde un punto de vista semántico, requieren necesariamente combinarse con un elemento que actúe como sujeto de las propiedades que denotan -lo cual diferencia a esta categoría de los nombres-, pero, al contrario de lo que sucede con otras categorías que requieren un sujeto -como la preposición-, no pueden tomar un complemento. Como es bien sabido, la presencia de complementos en el sintagma adjetival está siempre condicionada por la presencia de una preposición, por lo que se puede argumentar que el adjetivo es incapaz de introducir complementos por sí mismo.

Hale y Keyser notan que estas dos propiedades, la necesidad de combinarse con un adjetivo y la incapacidad de seleccionar por sí mismas un complemento, establecen requisitos contradictorios en la construcción de la configuración sintáctica. Siguiendo los principios de la estructura escueta de sintagma (Chomsky, 1993), el primer constituyente seleccionado por un núcleo ha de proyectarse necesariamente como su argumento interno o complemento; los sujetos, en la medida en que son especificadores estructurales, no pueden proyectarse como tales salvo que el núcleo que los seleccione contenga ya un complemento, pues los especificadores o argumentos externos solo se construyen cuando ya hay un primer constituyente que ha ocupado la posición de complemento. Por tanto, el adjetivo es problemático: no es capaz de introducir más que un constituyente, pero requiere que éste se proyecte en la posición de especificador. Parecidas observaciones se hacen en Baker (2003). 
La solución configuracional al problema es que el adjetivo se una parasíticamente a una categoría de naturaleza relacional que lo tome como complemento. En esta configuración, ilustrada en (18), la categoría de naturaleza relacional $-\mathrm{h} *_{\text {- }}$ toma al adjetivo como su complemento. De esta manera, el segundo constituyente que se combine sintácticamente con $\mathrm{h}^{*}$ se proyectará como especificador, lo cual es la posición canónica de los sujetos. Por esta razón, el adjetivo puede aprovechar parasíticamente la configuración de $\mathrm{h}^{*}$ para proyectar su sujeto.

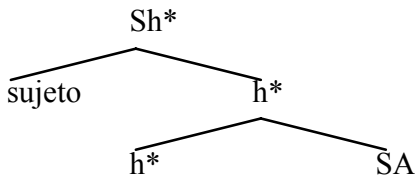

Sin embargo, esta estructura tiene un problema, y es que falta motivación semántica que explique la presencia de $h^{*}$. Obsérvese que la presencia de $h^{*}$ parece vacua desde el punto de vista del significado: introduce como uno de sus complementos a un elemento que, en apariencia, no establece con él ninguna relación semántica. Esto es un problema de gran calado, pues toda teoría gramatical -con independencia de la escuela desde la que procedase preocupa por encontrar las conexiones entre el significado y la estructura de una construcción.

Por tanto, parece necesario encontrar una correlación entre la estructura de $\mathrm{h}^{*}$ y una relación semántica conectada con el. Proponemos que h* es una instancia de lo que Bowers (1993, 2000) ha identificado como el Sintagma Predicación (SPred). Este sintagma es una estructura relacional que traduce, hasta cierto punto, lo que en lógica de primer orden se conoce como abstracción lambda. El SPred toma como complemento un predicado, esto es, una expresión semántica con una posición de variable, y la convierte en una predicación, al tiempo que introduce en su especificador el argumento que ese predicado requiere. De esta manera, si h* equivale al SPred, la relación semántica que buscamos queda satisfecha: el adjetivo es un predicado, pero, para convertirse en una predicación, requiere combinarse en la sintaxis con el SPred, que, respetando los principios de la estructura escueta de sintagma, puede introducir un sujeto como su especificador. La estructura, con su traducción semántica, queda representada en (19).

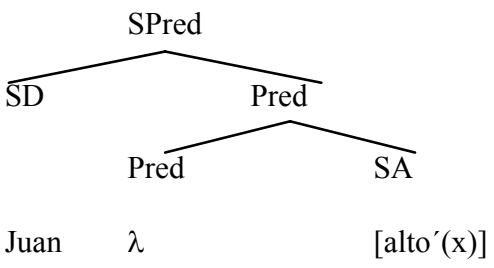

Queda por explicar qué rasgo convierte al adjetivo, léxicamente, en un predicado. Seguimos la propuesta de Spencer (1999) de que los adjetivos contienen un rasgo semántico Atr (Atribución) y la asociación entre el adjetivo y su argumento se establece coindizando 
en un nivel semántico -nosotros proponemos que se trata de la Forma Lógica (FL)- dicho rasgo con un rasgo semántico referencial que contienen todos los sustantivos. Detengámonos un momento en la implementación técnica propuesta por Spencer. En (20), el adjetivo alto tiene dos papeles temáticos que descargar: uno es el papel temático de paciente, $\mathrm{Pa}$, que queda saturado mediante ligado temático, y un papel semántico categorial (Atr), que se satura mediante identificación temática con el papel categorial del nombre, Ref. Representamos la identificación temática mediante un asterisco pospuesto.

(20) a. niño alto.

b. Ligado temático: $A<P a_{i}$, Atr $>, N<$ Referencia $_{i}>$

c. Identificación temática: $A<P a_{i}$, Atr* $^{*}, N<$ Referencia* $_{i}>$

Creemos que los papeles categoriales se pueden trasladar perfectamente a un lenguaje compuesto por rasgos, y, en este sentido, interpretamos la propuesta de Spencer como un procedimiento por el cual el rasgo referencial del nombre -el índice de identidad para Baker (2003)- se identifica en FL con el rasgo de atribución del adjetivo. Una vez incorporada esta implementación técnica a nuestra estructura, la representación es la de (21).

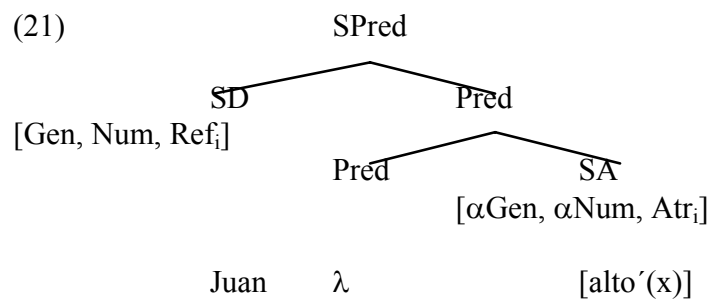

Así, la combinación del adjetivo con SPred, que le proporciona un sujeto de predicación, tiene varias funciones: le dota del argumento que requiere para satisfacer sus condiciones como predicado, lo convierte en una predicación y, en caso de que el argumento sea de la categoría adecuada y contenga género y número interpretables, le permite satisfacer sus rasgos de concordancia.

Recordemos que, anteriormente, hemos observado que en nuestra lengua existe una correspondencia biunívoca entre el establecimiento de una relación de concordancia y la elección del argumento que se convierte en el sujeto del adjetivo. Esta propiedad queda inmediatamente explicada si proponemos que la materialización léxica del núcleo Pred son los rasgos de concordancia del adjetivo. Esto queda representado en (22). 
(22)

$$
\begin{aligned}
& \text { SD Pred } \\
& \text { [Gen, Num, Refi } \mathrm{i}_{\text {Pred }} \\
& {\left[\alpha \mathrm{Gen}, \alpha \mathrm{Num}, \mathrm{Atr}_{\mathrm{i}}\right]} \\
& \text { Juan }-o \quad \text { alt- Lexicalización } \\
& \begin{array}{lll}
\text { Juan } & \lambda \quad\left[\text { alto' }^{\prime}(\mathrm{x})\right] \mathrm{FL}
\end{array}
\end{aligned}
$$

Nótese que estos dos procesos -establecimiento de concordancia e identificación argumental- son lógicamente independientes, aunque, en una situación como la de (22), suceden simultáneamente como resultado de la misma estructura. Argumentaremos que en el caso de los adverbio en -mente, estos procesos suceden de forma separada, porque el sujeto de la predicación es un elemento que posee rasgos de género y número, pero no un rasgo referencial. Por ello, puede satisfacer la concordancia del adjetivo, pero no sus requisitos como predicado.

Como se habrá advertido ya, es casi inevitable establecer una comparación entre este SPred, lexicalizado como rasgos de concordancia y cuya función es la de introducir el sujeto del adjetivo, con el Sintagma Tiempo en el dominio verbal, cuya manifestación estándar son también rasgos de concordancia y que, igualmente, permite proyectar al sujeto en su especificador. La comparación con el dominio verbal no termina aquí: seguimos la propuesta de Kayne (1994) de que la estructura predicativa de los adjetivos está dominada por un Sintagma Complementante que convierte la estructura predicativa en una proposición. Evidencia independiente de que esta proyección existe en espanol con los adjetivos la encontramos, por ejemplo, en Rafel (2000).

La última proyección de nuestra estructura es el Sintagma Grado (SG), del que ya hemos hablado previamente en las secciones anteriores. Nuestra propuesta es que esta proyección se encuentra entre la estructura de predicación y el adjetivo. La motivación de este orden de proyecciones es fundamentalmente la composicionalidad semántica. Consideremos la oración de (23).

\section{(23) Juan es altísimo.}

La interpretación de esta oración es que la propiedad de ser alto se manifiesta en un valor muy alto, y Juan posee dicha propiedad; su semántica no es que la relación que se establece entre Juan y la propiedad de ser alto aparezca en un alto grado. Dada esta interpretación semántica, y suponiendo, como es estándar, que las relaciones jerárquicas en la sintaxis motivan las relaciones semánticas que se establecen en una estructura, resulta necesario proponer que el SG está jeráquicamente más alto que el SA, pero más bajo que el SPred, pues la noción semántica introducida por el grado tiene ámbito sobre la propiedad denotada por el adjetivo y no sobre la estructura de predicación introducida por Pred. 


\subsubsection{Concordancia $y-$ mente}

Hemos aportado pruebas, tomadas de Iglesias Bango y Rodríguez Ramalle, de que la aportación de -mente en la estructura de la palabra es semánticamente vacua, dado que no altera las propiedades introducidas por el adjetivo. Nuestra propuesta es apoyarnos en esta defectividad semántica para derivar las propiedades de los adverbios en -mente y relacionarlos con la estructura general de los adjetivos predicativos.

Supongamos que en la posición de sujeto del adjetivo, el especificador de SPred, se proyectara un constituyente que carece de un rasgo referencial pero contiene rasgos de género y de número interpretables. En esta situación, la correspondencia entre concordancia y predicación se rompería, dado que este constituyente sería capaz de satisfacer los rasgos de género y número del adjetivo, pero no podría actuar como su predicado. La estructura resultante sería la de (24).

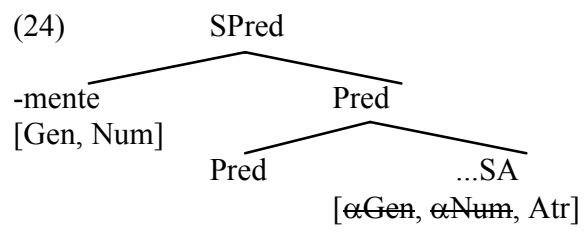

La presencia de -mente satisface parte de las propiedades del adjetivo, pero no aquellas que tienen que ver con su naturaleza de predicado. Por esta razón, el adjetivo sigue requiriendo identificar su rasgo Atr con algún otro rasgo interpretable. En una configuración adecuada, el adjetivo identificará el evento verbal, el acto de habla o cualquier otro elemento que sea del tipo semántico adecuado.

Esto explica que la gramática emplee los adjetivos en -mente como una estrategia para obtener predicados que tomen categorías no nominales. La presencia de -mente introduce una forma de satisfacer la concordancia de género y de número, pero no satura al adjetivo como predicado, de manera que este elemento puede asociarse semánticamente a un constituyente que, por no tener el tipo adecuado de rasgos, no hubiera podido funcionar como su sujeto.

\subsection{Obtención del orden de los morfemas}

Dada nuestra estructura, obtenemos de forma directa el orden de los morfemas en el interior de los adverbios en -mente y podemos, además, explicar los dos patrones de coordinación que se constatan en las distintas lenguas romances. La única operación que requerimos es el movimiento de núcleos, que se halla sujeto a condiciones de localidad estricta, y, por lo que parece, es una operación que sigue siendo necesaria en el marco sintáctico actual (Pesetsky y Torrego, 2001; Matushansky, 2006, entre otras muchas referencias). Recordemos, en aras de la exposición, la estructura sintáctica que hemos motivado previamente (25). 
(25)

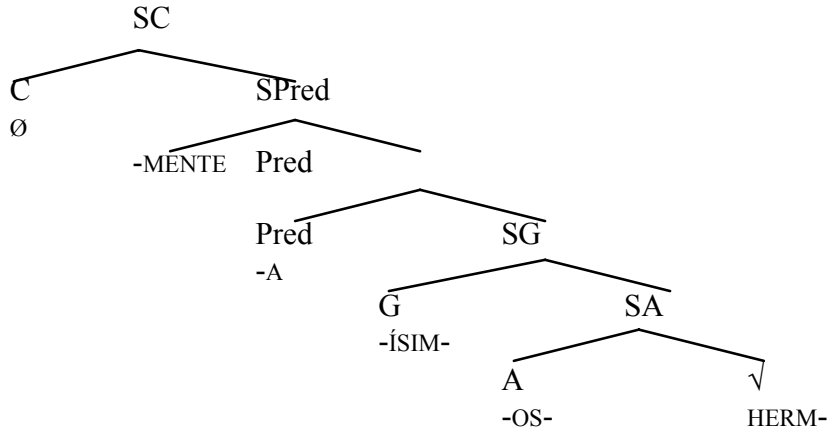

El movimiento cíclico de cada uno de los núcleos hasta el núcleo de la proyección que le domina inmediatamente da como resultado lo que en la terminología anglosajona se ha dado en denominar snowball movement, una serie de movimientos en que se obtiene un orden de morfemas que refleja, especularmente, el orden de las proyecciones de las que dichos morfemas eran núcleos. En efecto, siguiendo los supuestos básicos de la teoría del movimiento de núcleos, cada núcleo se mueve a una posición de adjunto del núcleo superior (26); combinando esta configuración con el Axioma de Correspondencia Lineal de Kayne (1994), dado que un adjunto no es mandado-c por la proyección con la que se combina, se sigue que un núcleo que se adjunta a un núcleo superior se linearizará a la izquierda de éste.

(26)

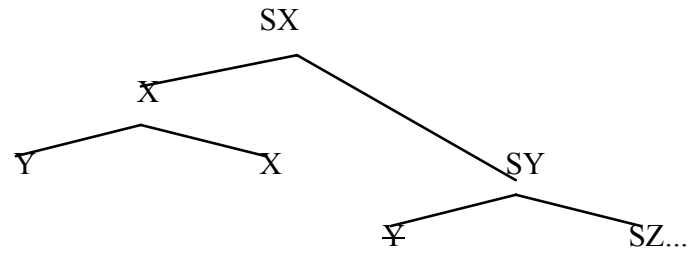

La raíz se mueve a $\mathrm{A}$, donde se adjunta; ambos constituyentes se mueven a $\mathrm{G}$; el conjunto formado por estos elementos se desplaza a Pred; finalmente, toda esta estructura se mueve hasta C. De esta manera obtenemos el orden de (27).
(27) $[[[[[$ herm- $]$
os- $]$
A
$\begin{array}{ll}\text { isim- }] & a-1 \\ G & \text { Pred }\end{array}$
$a-]$
Pred
$\varnothing-]$
[-mente]
$\sqrt{ }$ A $G$ Pred $C$ Gen., Num.

Nótese que en nuestra propuesta es crucial que el movimiento de núcleos tenga lugar en la sintaxis, al igual que en las propuestas de los autores que hemos mencionado anteriormente. Esta idea se enfrenta a lo propuesto a menudo en el marco de la Morfología Distribuida (cfr. especialmente Embick y Noyer, 2001), donde se sugiere que esta clase de movimientos se producen en un nivel post-sintáctico, debido a propiedades morfofonológicas de las piezas que materializan los núcleos. Aparte del problema teórico al que da lugar admitir la existencia de operaciones post-sintácticas en una estructura 
sintáctica, obsérvese que es crucial que el adjetivo, con toda la información que contienen los núcleos implicados, se desplace a la proyección más alta de (25). Esto se debe a que, en un adverbio predicativo de este tipo, requiere tomar un argumento. Dicho argumento es el evento contenido en el SV al que se adjunta, o la función que denota una proposición en el SC. De aquí se sigue que estos rasgos de SC o SV deben ser accesibles para el adjetivo, y esto no es posible, por localidad, si éste se encuentra en alguna posición más baja que el núcleo $\mathrm{C}$, que proyecta y se fusiona como adjunto.

Los principios sintácticos que restringen el movimiento de núcleos también explican la posición periférica de -mente, pese a encontrarse en un nudo intermedio del árbol. El movimiento de núcleos solo se produce entre un núcleo y su complemento, y es imposible aplicarlo entre el núcleo y su especificador. Dado que -mente está en posición de especificador, predecimos que se realizará periféricamente.

Esto explica por qué en los procesos de coordinación de dos o más adverbios en -mente solo se materializa uno de ellos (el primero o el último, dependiendo de la variedad de la que estemos hablando). Como es normal, un rasgo de género o de número interpretable, al no tener que borrarse, puede concordar con tantos elementos como aparezcan en la derivación; por ello, varios adjetivos coordinados en el interior de la estructura del adverbio pueden compartir un solo -mente. Si esto es así, obtendríamos una estructura como la siguiente, donde no representamos los nudos que no son relevantes para nuestra explicación $(28)^{2}$.

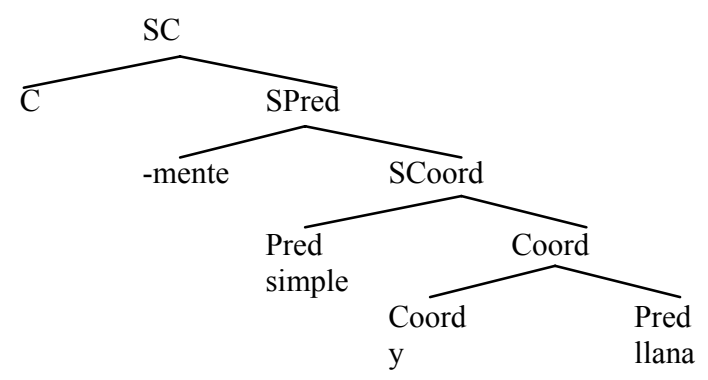

A partir de esta misma estructura, podemos derivar tanto el orden en que el último adjetivo es el que aparece adyacente a -mente, igual que en el castellano contemporáneo, como aquellas variantes, históricas o dialectales, en que -mente sigue al primer adjetivo. Esto depende de qué estructura es la que sea atraída al SC.

Si el elemento que se desplaza al SC es todo el SCoord, obtenemos el orden en que mente sigue a todos los adjetivos.

\footnotetext{
${ }^{2}$ Como se ve, en esta estructura admitimos que es posible la coordinación de proyecciones no máximas. Cfr. Camacho (2003).
} 
(29)

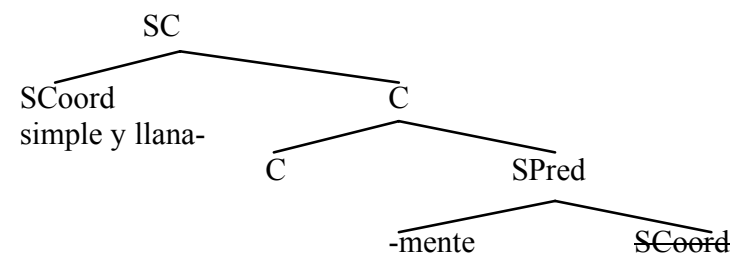

Por el contrario, si solo se desplaza el primer especificador de la estructura coordinada, el orden es aquél en que -mente aparece tras el primer elemento coordinado.

(30)

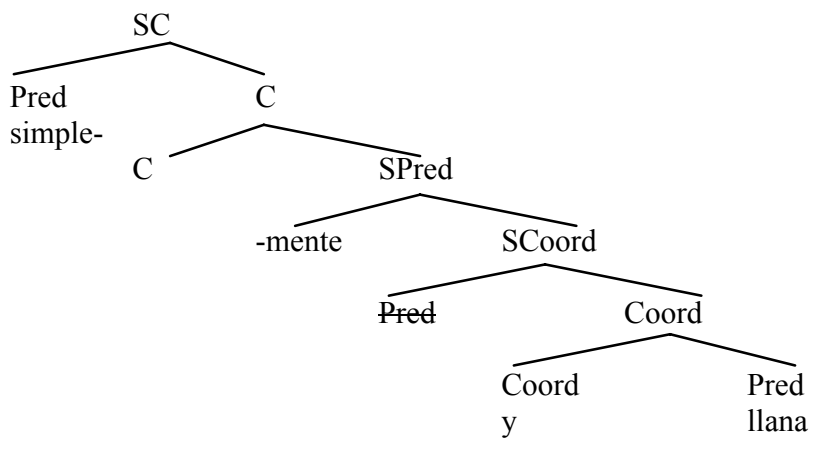

Por tanto, la diferencia entre las lenguas en las que -mente se combina con el primero o el último adjetivo depende exclusivamente de qué rasgo categorial es el que es atraído por $\mathrm{SC}$.

\subsection{Por qué mente}

Otros autores, como Kornfeld (2006), han propuesto que -mente lexicaliza un rasgo sintáctico 'orientado al verbo'. Nosotros, por nuestra parte, a la luz de los casos en que mente no se orienta al verbo o se combina con un adjetivo no predicativo, hemos propuesto que lexicaliza rasgos interpretables de género y número. Mente es, principalmente, un sustantivo del español contemporáneo, y nuestro análisis explica que la palabra escogida sea un elemento que pertenece la clase de los sustantivos. No obstante, aún no hemos explicado por qué no se pueden emplear otros sustantivos para construir adverbios de esta clase.

Sin ninguna duda, diversos factores históricos con una motivación semántica rastreable han hecho que en el castellano contemporáneo se emplee la forma -mente para materializar una matriz de rasgos con género y número, pero carente de la capacidad de ser un sujeto de predicación. No es este el lugar de revisar estos factores; sin embargo, querríamos contribuir a la comprensión de esta forma proporcionando una formalización explícita que indique por qué, en las variantes contemporáneas, el único sustantivo que puede materializar esta matriz es -mente, y no otro. 
Supongamos que la inserción de las piezas de vocabulario se produce como resultado de la competición entre distintas formas que, en principio, podrían materializar el mismo nudo. Esto ha sido propuesto es numerosos estudios (cfr., por ejemplo, Bonet, 1991; Noyer, 1992; Halle, 1997) que tratan de explicar por qué no se produce una relación biunívoca entre los rasgos sintácticos y los morfemas que los materializan. Todos estos estudios están de acuerdo en que, cuando es posible insertar varios elementos distintos en el mismo nudo, uno de ellos, aquel cuyas propiedades se aproximan de manera más exacta a las del nudo sintáctico, es el que vence. Es decir, si una pieza léxica tiene todas las propiedades menos una, y existe otra pieza léxica que tenga exactamente las mismas propiedades que el nudo sintáctico, el candidato óptimo será el segundo.

Por tanto, si aceptamos esta perspectiva de los hechos, una propuesta formal que explicaría por qué en este contexto sólo es posible insertar-mente sería la de asociar esta pieza de vocabulario con los rasgos representados en (31). Compárese esta representación a la entrada de (32), que comparten todos los sustantivos.

$$
\begin{array}{lll}
\text { MENTE } & \leftrightarrow & \begin{array}{l}
\text { [Gen, Num, Ref] } \\
\text { [Gen, Num] }
\end{array} \\
& & \\
\text { CASA, PERRO, PLATO... } & \leftarrow \rightarrow & \text { [Gen, Num, Ref] }
\end{array}
$$

La interpretación de (31) es la siguiente: la pieza léxica mente está asociada a dos posibles matrices de rasgos. La primera de ellas está compartida por todos los sustantivos de la lengua: género, número y un rasgo de referencia que le permite ser sujeto de predicación. Por tanto, en un contexto nominal, esta pieza competirá con todos los demás sustantivos, cuya entrada es la de (32). La segunda matriz de rasgos es exclusiva de mente, que, en este contexto, está asociada exclusivamente a género y número. Ningún otro sustantivo comparte esta segunda estructura de rasgos, por lo que en un contexto adverbial el candidato óptimo va a ser siempre mente, dado que no contiene ningún rasgo que no aparezca en el nudo sintáctico que va a ser lexicalizado.

\section{Productividad: por qué se dice lo que se dice y no se dice lo que no se dice}

Parte del atractivo inicial de una teoría derivacional de los adverbios en -mente es que, al enmarcarse dentro del Lexicalismo, la productividad limitada de estas formas se convierte en un hecho que deriva de las propiedades idiosincrásicas del léxico. En general, se suele admitir que los procesos sintácticos son máximamente productivos, mientras que las operaciones léxicas están sometidas a menudo a las particularidades e irregularidades de los elementos implicados en una operación. La observación de Scalise acerca de que no todo adjetivo puede dar lugar a un adverbio en -mente muestra se ha tomado como un argumento en contra de quienes quieren tratar el proceso como un caso de flexión y, por implicación, puede entenderse como un argumento contra un análisis sintáctico. En esta sección mostraremos que aquellos adjetivos que no pueden dar adverbios en -mente poseen un significado que es incompatible con la configuración sintáctica. 


\subsection{Adjetivos relacionales y otros adjetivos no predicativos}

En ocasiones se ha afirmado que los adjetivos relacionales, como político, económico o cinematográfico, son incapaces de dar adverbios en -mente debido a su incapacidad para denotar cualidades de los objetos. Una teoría en la que la presencia de -mente satisfaga alguna de las propiedades semánticas del adjetivo, sea la de sujeto de predicación o la materialización de un rasgo de significado del adjetivo, es incapaz de explicar la existencia de formaciones como las de (33), donde encontramos lo que se ha llamado en ocasiones un adverbio de punto de vista.

(33) a. Esta legislatura ha sido políticamente un desastre.

b. Cinematográficamente, estamos ante uno de los pilares del S.XX.

En estos adverbios, la relación expresada por el adjetivo de la base toma a la situación (33a) o a la proposición completa (33b) como término: la situación de la primera oración posee una propiedad determinada si se considera en su relación con la política y la proposición de (33b) es verdadera en la medida en que se relaciona con el cine.

Nuestra teoría predice la existencia de esta clase de adverbios, porque la presencia de mente no desempeña directamente ningún papel semántico, sino que se ocupa de satisfacer la concordancia del adjetivo y los adjetivos relacionales concuerdan tanto como los predicativos. Si un adjetivo relacional establece una relación semántica con una categoría no nominal, como los eventos, las situaciones o las proposiciones, se verá con los mismos problemas que un adjetivo calificativo, pues dichas categorías no podrán satisfacer su concordancia. La presencia de -mente, como en el caso anterior, soluciona este problema.

El mismo razonamiento nos permite explicar por qué otros adjetivos no predicativos, como los de (34), pueden dar adverbios de esta clase: pese a compartir muy poco de su semántica con los adjetivos calificativos, su forma es la misma en la medida en que deben concordar.

(34) a. meramente, mismamente

b. supuestamente, presuntamente

c. constantemente, continuamente

d. primeramente, últimamente

Ninguno de estos adjetivos es propiamente calificativo. (34a) son adjetivos intensionales; (34b), epistémicos; (34c) presenta adjetivos adverbiales que denotan una noción relacionada con el aspecto externo, y (34d) es un adjetivo ordinal. Ninguno de estos elementos designa propiedades que puedan ser intersectivas con la denotación del nombre, $\mathrm{y}$, sin embargo, pueden dar adverbios en -mente, lo cual confirma nuestro análisis formal en que la presencia de este constituyente se debe a la existencia de flexión. 


\subsection{Propiedades físicas y propiedades de las proposiciones}

En una estructura sintáctica, el significado de una construcción es una función del significado de cada uno de los constituyentes y de la configuración en la que se encuentran. De aquí se sigue que no existe ninguna necesidad lógica de que la ausencia de adverbios en -mente formados a partir de ciertos adjetivos derive de una idiosincrasia propia de las operaciones léxicas. Otra posibilidad lógica sería que el significado de ciertos adjetivos no sea compatible con un sujeto eventivo o proposicional, o bien que el tipo de proyección a la que se une el adverbio es incompatible con ciertos aspectos del significado del adjetivo.

Desde numerosas corrientes se diferencian dos tipos distintos de significado, que, de forma general, pueden caracterizarse como el significado que se deriva de las distintas estructuras que ordenan una construcción -que, en cierta medida, es predecible y generalizable- y el que se aprende con cada una de las piezas que intervienen en esa construcción -que no siempre es sistemático-. La distinción tiene distintos nombres en distintas corrientes (semántica estructural vs. semántica conceptual en la Morfología Distribuida, semántica sintáctica vs. semántica difusa en el trabajo de Michal Starke, el esqueleto semántico vs. los conceptos en Lieber, 2004, etc.), pero todas las propuestas se ven forzadas a proponer, en cierto punto, que existen entradas léxicas en las que las palabras se asocian a un significado no predecible. Utilizaremos este tipo de entradas para dar cuenta de la productividad de los adverbios en -mente con adjetivos que designan propiedades físicas.

Entre los adjetivos de propiedades físicas, solo un subconjunto de ellos pueden dar adverbios en -mente. Los adjetivos que se encuentran bajos en la jerarquía funcional, y, por tanto, no admiten implican necesariamente un juicio por parte del hablante -al contrario que hermoso-, no tienen esta misma productividad (35).

(35) *rojamente, *amarillamente; *redondamente, *cuadradamente...

En realidad, el problema no parece ser que ciertos adjetivos de propiedad física no puedan dar adverbios en -mente, sino, más bien, que ciertos adjetivos de esta clase admiten también una lectura en la que la dimensión expresada se reinterpreta como una escala de intensidad, de duración o de otras propiedades generales de los eventos. Un adjetivo como alto $\mathrm{u}$ hondo, cuando forma adverbios en -mente, no se refiere a la altura o a la hondura física (36).

(36) a. Hablar altamente (=hablar en estilo elevado)

b. Preocuparse hondamente (=preocuparse con intensidad)

Proponemos que, tal vez debido a su posición en la jerarquía funcional (Cinque, 1999), los adjetivos de (35) y de (36) tienen entradas de semántica conceptual diferentes. Adjetivos como alto designan escalas que pueden reinterpretarse metafóricamente como escalas no físicas, mientras que otros, como rojo, solo admiten escalas físicas. En el primer grupo de adjetivos, dependiendo de la naturaleza de la entidad de la que se predique el adjetivo, el valor de verdad se evaluará con respecto a una u otra escala (37). 
(37) alto' (individuo): el tamaño del eje vertical es mayor o igual que un valor estándar.

(evento) = la consideración moral o estética de un acto es mayor o igual que un valor estándar.

Los adjetivos de color o de forma están asociados fuertemente con las entidades físicas (Quine, 1960), por lo que es plausible que en sus entradas solo encontremos solamente escalas físicas (38)

(38) amarillo' (individuo) $=$ el color es $\mathrm{X}$. (individuo) $=$ la raza es $\mathrm{Y}$.

Por tanto, si el adjetivo se encuentra en una configuración en que se predica de un evento o de una proposición, la estructura resultante será agramatical precisamente porque no será posible computar el valor de verdad de una propiedad como la de (38) aplicada a un evento o a una proposición. Por tanto, con un análisis como este, queda patente que la inexistencia de formaciones como rojamente puede derivarse de condiciones semánticas independientes que pesan sobre las estructuras sintácticas.

Por la misma razón que ciertos adjetivos no admiten ninguna lectura si no se predican de individuos, esperamos que otros adjetivos, cuando den adverbios en -mente, solo puedan desempeñar una función dentro de la cláusula. Esperamos que adverbios como francamente, sinceramente o desgraciadamente sean únicamente adverbios orientados al enunciado o a la enunciación, porque adjetivos como franco, sincero o desgraciado solamente puedan predicarse de proposiciones. De manera semejante, esperamos que los adjetivos relacionales solo puedan ser adverbios de punto de vista o de dominio (Kovacci, 1999), porque los adjetivos sobre los que se construyen expresan relaciones y denotan clases de individuos. Los estudios de Iglesias Bango (2004) o de Rodríguez Ramalle (2001) a los que aludíamos antes proporcionan pruebas adicionales de que la semántica del adjetivo determina su función como adverbio $\mathrm{y}$, por tanto, puede explicar la imposibilidad de tener función adverbial.

\section{Conclusiones}

En este trabajo hemos defendido un análisis sintáctico de los adverbios en -mente en el que hemos explicado a partir de la misma estructura las propiedades que estas formas poseen con respecto a su posición, su relación con los adjetivos, el orden de sus formantes, la existencia de concordancia interna, la particular naturaleza de la forma -mente y su productividad morfológica. Hemos tomado partido por una teoría de la morfología en la que la formación de palabras sucede en la sintaxis, de tal manera que los morfemas se representan separadamente en la sintaxis y las palabras se construyen mediante movimiento de núcleos.

Con respecto a otros estudios que han analizado los adverbios en -mente como el resultado de operaciones sintácticas, como el de Zagona (1990) o Kornfeld (2006), nuestra 
propuesta presenta la novedad de que la estructura del adverbio en -mente está integrada con la estructura del adjetivo. Nuestro análisis, frente a estas dos propuestas, defiende asimismo que la única función de la forma -mente es la de satisfacer la concordancia del adjetivo, con lo cual, frente a la propuesta de estas autoras, predecimos que los adjetivos relacionales o no predicativos pueden ser bases para la formación de estos adverbios. De igual manera, nuestra estructura predice datos como los encontrados en Iglesias Bango (2004) y Rodríguez Ramalle (2002), donde se muestra que la función del adverbio es un reflejo directo de las propiedades semánticas que posee el adjetivo que se halla en su base. La variabilidad de funciones de un adverbio en -mente hace imposible analizar estas piezas como el resultado de combinar un adjetivo con un rasgo del tipo de 'orientado al verbo', como se propone en Kornfeld (2006); nuestro trabajo, en cambio, permite dar cuenta de esta variabilidad.

En un sentido más general, de forma indirecta y en la medida en que este análisis se pueda mantener, nuestro trabajo aporta una pieza más que va en la dirección general de que el adverbio no existe como una estructura sintáctica especial y no es más que una etiqueta descriptiva que se refiere a distintos tipos de entidades que desempeñan la función de modificadores, adjuntos o complementos circunstanciales.

\section{Referencias bibliográficas}

Alarcos, E. (1951): Gramática estructural. Madrid, Gredos.

Alcina, J. y J. M. Blecua (1975): Gramática española. Barcelona, Ariel.

Alonso, A. y P. Henríquez Ureña (1955): Gramática castellana. Barcelona, Vox.

Arad, M. y U. Shlonsky (2002): Affixation above and below VP. Conferencia impartida en el MIT Workshop.

Baker, M. (2003): Lexical categories. Cambridge, Cambridge University Press.

Bartra, A. y A. Suñer (1997):'Inert agreement projections and the syntax of bare adjectives", Probus , 9: 1, págs. 1-34

Bello, A. (1847): Gramática de la lengua castellana destinada al uso de los americanos. Edición crítica de Ramón Trujillo (1981). Tenerife, Instituto de Lingüística Andrés Bello.

Bonet, E. (1991): Morphology after syntax. Pronominal clitics in Romance. Tesis doctoral, MIT.

Bosque, I. (1989): Las categorías gramaticales. Relaciones y diferencias. Madrid, Síntesis.

Bowers, J. (1993): “The syntax of predication”, Linguistic Inquiry, 24: 4, págs. 591-656.

Bowers, J. (2001): "Predication". En M. Baltin y C. Collins (eds.), The Handbook of Contemporary Syntactic Theory. Oxford, Blackwell, págs. 299-333.

Bybee, J. (1985): Morphology: A Study of the Relation between Meaning and Form. Amsterdam, John Benjamins.

Caha, P. (2007): The superset principle. Ms., Universitetet i Tromsø.

Camacho, J. (2003): The structure of Coordination. Conjunction and Agreement Phenomena in Spanish and Other Languages. Berlin, Springer.

Chomsky, N. (1995): "Bare Phrase Structure". En G. Webelhuth (ed.), Government and Binding Theory and the Minimalist Program. Oxford, Blackwell Publishers, págs. 383-439.

Chomsky, N. (1998): Minimalist Inquiries: The Framework. MIT Working Papers in Linguistics 15.

Chomsky, N. (2000): "Derivation by phase". En M. Kenstowicz, (ed.), Kenneth Hale: A Life in Language. Cambridge (Mass.), MIT Press, págs. 1-52. 
Chomsky, N. (2001): Beyond Explanatory Adequacy, MITWPL.

Cinque, G. (1999): Adverbs and functional heads. A cross-linguistic perspective. Oxford, Oxford University Press.

Corbett, G. (1987): "The morphology/syntax interface: evidence from possessive adjectives in Slavonic", Language, 63: 2, págs. 299-345.

Corver, N. (1997): "The internal syntax of the Dutch extended adjectival projection", Natural Language and Linguistic Theory, 15: 3, págs. 289-368.

Cresswell, M. (1976): “The semantics of degree”. En B. Partee (ed.), Montague grammar. New York, Academic Press, págs. 261-292.

Eguren, L. y O. Fernández Soriano (2004): Introducción a una sintaxis minimista. Madrid, Gredos.

Embick, D. y R. Noyer (2001): "Movement Operations after Syntax", Linguistic Inquiry, 32: 4, págs. 555-598.

Fábregas, A. (2005): La definición de la categoría gramatical en una morfología orientada sintácticamente. Tesis doctoral, Universidad Autónoma de Madrid / Instituto Universitario de Investigación Ortega y Gasset.

Hale, K. y S. J. Keyser (1993): "On argument structure and the lexical representation of syntactic relations". En K. Hale y S. J. Keyser (eds.), The View from Building 20, Cambridge (Mass.), MIT Press, págs. 53-110.

Hale, K. y S. J. Keyser (2002): Prolegomenon to a Theory of Argument Structure. Cambridge (Mass.), MIT Press.

Halle, M. (1997): "Distributed morphology: impoverishment and fission”. En B. Bruening et alii (eds.), Papers at the Interface. MITWPL 30, Cambridge (Mass.), MIT, págs. 425-449.

Hjelmslev, L. (1928): Principes de grammaire génerale. Copenhaguen: Bianco Lunos Boktrykkeri.

Iglesias Bango, M. (2004): "El comportamiento sintáctico de los adverbios terminados en -mente". En M. Villayandre Llamazares (ed.), Actas del V Congreso de Lingüística General, vol. 2, Madrid, Arco Libros, págs. 1633-1652.

Kamp, H. (1975): "Two theories about adjectives". En E. Keenan (ed.), Formal semantics of Natural Language, Cambridge, Cambridge University Press, págs. 123-155.

Kayne, R. (1994): The Antisymmetry of Syntax. Cambridge (Mass.), MIT Press.

Kennedy, C. (1999). Projecting the adjective: The syntax and semantics of gradability and comparison. New York, Garland.

Kornfeld, L. (2006): Romance adverbs in -mente and the relation morphology-syntax. Comunicación presentada en el XVI Coloquio de Gramática Generativa, Madrid, abril de 2006.

Kovacci, O. (1999): "El adverbio". En I. Bosque y V. Demonte (dirs.), Gramática descritpiva de la lengua española. Madrid, Espasa, págs. 705-786.

Lapointe, S. (1979): A Theory of Grammatical Agreement. Tesis doctoral, Amherst, University of Massachusetts.

Lieber, R. (2004): Morphology and Lexical Semantics. Cambridge, Cambridge University Press.

Lenz, R. (1935): La oración y sus partes. Madrid, Revista española de filología.

Marantz, A. (2001): Words. Ms., MIT.

Matushansky, O. (2006): "Head movement in linguistic theory", Linguistic Inquiry, 37: 1, págs. 69109.

Noyer, R. (1992): Features, positions and affixes in Autonomous Morphology. Tesis doctoral, Cambridge (Mass.), MIT.

Pesetsky, D. y E. Torrego (2001): “T-to-C: Causes and Consequences”. En M. Kenstowicz (ed.), Ken Hale: A Life in Language. Cambridge (Mass.), MIT Press, págs. 355-426.

Quine, W. V. (1960): Word and object. Cambridge (Mass.), MIT Press. 
Rafel, J. (2000): Complex Small Clauses, Tesis doctoral, Barcelona, Universidad Autónoma de Barcelona.

Rainer, F. (1993): Spanische Wortbildungslehre. Tübingen, Niemeyer.

Ramchand, G. (2008): First phase syntax. Cambridge, Cambridge University Press.

Rodríguez Ramalle, T. (2002): "Los adverbios de manera como predicados de los subeventos verbales", $R E L, 31$ : 1, págs. 107-143.

Scalise, S. (1993): Morfologia generativa, Bolonia, Il Mulino.

Seco, M. (1972): Gramática esencial del español. Madrid, Aguilar.

Spencer, A. (1999): "Transpositions and argument structure". En G. Booij y J. van Marle (eds.), Yearbook of Morphology 1998. Dordrecht, Kluwer, págs. 73-102

Varela, S. (1990): Fundamentos de morfología, Madrid, Síntesis.

Zagona, K. (1990): “-Mente adverbs, compound interpretation and the projection principle", Probus, 2, págs. 1-30.

Zwaarts, J. (1992): $X^{`}$-Syntax $X^{\prime}$ 'semantics. On the Interpretation of Lexical and Functional Heads. Utrecht, OTS. 